\title{
SPATIAL DATABASE UPDATING USING ACTIVE CONTOURS FOR MULTISPECTRAL IMAGES: APPLICATION WITH LANDSAT7
}

\author{
Sylvie Jodouin, Layachi Bentabet, Djemel Ziou, Jean Vaillancourt \\ Départment de Mathématiques et d'Informatique \\ Université de Sherbrooke, Canada \\ \{jodouin, bentabet, ziou, vaillanc\}@DMI.Usherb.CA
}

KEY WORDS: Active contours, snakes, multi-spectral imagery, spatial data integration.

\begin{abstract}
This paper presents a fully automated system for area detection based on satellite images and topographic features from a database. The surfaces issued from the database are refined using active contours (also called snakes) according to updated information provided by the multi-spectral images. The snake is reformulated using finite elements, allowing for the processing of smaller structures thus low time processing. We propose a new external energy measure through a formula by combining statistical and radiometry features. Usually the snake model is controlled by various parameters that are difficult to set by a trial-and-error process. We propose an automatic setting of these parameters and an update of the snake topology. The proposed updates allow the snake to be deformed in order to provide accurate localization. Our approach is validated in a series of tests on LANDSAT7 images.
\end{abstract}

\section{INTRODUCTION}

Spatial databases are gaining popularity as a reference tool in many fields of application. The spatial information providers are currently concerned by how to maintain data updated and also how to increase its accuracy with limited resources. This problem could be overcome through image processing techniques, which allows increasing accuracy of both geometric and temporal features.

An increasing number of methods to update spatial information based on image processing began to appear in the latest years. From the proposed methods, we are mostly interested with those based on the snakes. The proposed approaches deal with different type of images, mainly with single band images issued from satellite or airborne and from different type of sensors such as radar or optical (Auclair-Fortier et al 2001, Bentabet et al 2001, Horritt, 1999). The snake model is defined according to the geometry of the target feature. Some works present linear snakes to search linear features such as roads (Auclair-Fortier et al 2001, Bentabet et al 2001) and others present closed snakes to obtain a description of area features such as water regions (Horritt, 1999).

In this paper, we propose a method to update existing area features from a given spatial database by the use of multi-spectral images. The available database vectors provide an interesting initialization for the area localization process. In this context, the closed snake approach was presented as a natural solution. The first contribution of this work is the formulation of the external energy of the snake that combines statistical and radiometry information. Secondly, the snake parameters are fixed automatically from the spatial database and are updated during the localization process. Finally, an efficient implementation of the snake using finite elements provides accurate localization with low time processing.

\section{APPROACH}

A snake is defined as a parametric curve $v(x, y)=v(x(s, t), y(s, t))$ which is allowed to deform from some arbitrary initial location within an image towards the desired final location. Thus, the use of snakes involves a two steps process being an initialization and the iterative minimizing process. To initialize the snake, we first perform the 
discretization of the database vectors. This operation is performed using a finite element method together with the approximation of the snakes with Hermite polynomials. The final snake location is obtained through minimization process acting upon an internal and an external energy function defined as follows (Kass et al 1988).

$$
E_{\text {tot }}(t)=E_{\text {int }}(t)+E_{\text {ext }}(t)
$$

where $E_{\text {ext }}(t)$ is the external energy, $E_{\text {int }}(t)$ the internal energy. As described in classical snake modeling approach (Auclair-Fortier et al 2001, Bentabet et al 2001, Horritt, 1999, Kass et al 1988), the internal energy acts as a stabilizer to the external data irregularities. The standard internal formulation is given as follow:

$$
E_{\text {int }}(t)=\oint\left(\alpha\left|v^{\prime}(s, t)\right|^{2}+\beta\left|v^{\prime \prime}(s, t)\right|^{2}\right) d s
$$

where $\alpha$ and $\beta$ are respectively the elasticity and rigidity parameters. In this paper, we will elaborate in more detail about the external energy and the setting of the snake parameters.

\section{EXTERNAL ENERGY}

The external energy represents the image forces $E_{\text {ext }}(t)$ attracting the snake. This energy is calculated as follow:

$$
E_{e x t}(t)=\oint F_{e x t}(v(s, t)) d s
$$

where $F_{\text {ext }}(v(s, t))$ is the calculated force attracting the snake towards the boundary of the region. In our case, this force is defined through a combination of radiometry and statistical information. The radiometry feature is obtained from a multi-band edge detector (Drewniok, 1994). This detector takes as input all bands of the multispectral image and provides a single edge map.

The statistical properties of the targeted area are computed assuming the regions of interest being normally distributed. If we consider a population given by the pixels within the initial area provided by the database through $\mathbf{n}$ bands, the mean vector and the covariance matrix will be defined as follow:

$$
\mathrm{m}=E\{\mathrm{x}\} \text { and } C=E\left\{(\mathrm{x}-\mathrm{m})(\mathrm{x}-\mathrm{m})^{T}\right\}
$$

where $\mathbf{x}$ is a radiometry vector of dimension $\mathbf{n}, \mathrm{E}\{\mathbf{a r g}\}$ is the expected value of the argument, $T$ indicates vector transposition. The dimension of the matrix $C$ is $\mathbf{n} \times \mathbf{n}$. Considering the radiometry and statistical information issued for the image, we observe the advantages and drawbacks of each feature. Indeed, the edge detector allows accurate location of area boundaries, but gives false edges for textured regions and week response inside the region. The statistical feature, it provides a good model for textured regions, but week localization of area boundaries. Within the actual framework, the two features are complementary in their strength and weakness. Indeed, the statistical information gives good information inside the region compared to the edge map that provides accurate location of the region limits when we get closer to the contour. Considering these facts, the external forces applied on the snake are obtained by a weighted combination of both statistical and radiometry based values. For clarity in the following formulas, we will work for a given time $t=t_{0}$ and omit this time argument. The external force at the curvilinear abscise on the snake is defined as follows:

$$
F_{\text {ext }}(v(s))=\left(\max \left(\left(1-\frac{\operatorname{Grad}_{(v(s))}}{\operatorname{Grad}_{\text {mean }}}\right), 0\right)\right)^{\tau} e^{-(1-\tau) \cdot \operatorname{Dist}(v(s))}
$$


where $\tau \in[0,1]$ is a calculated weighting combination value, $\operatorname{Grad}(v(s))$ is the multi-band edge detector response, Grad $_{\text {mean }}$ is the mean value of the gradient evaluated for the specific type of region according to the hole image. The $\operatorname{Dist}(v(s))$ evaluates the proximity of a pixel to the statistical properties of the region. From this formulation, we can observe that when a measure of the external energy is taken inside the region, the first term of equation (6) (radiometry measure) is close to one. Thus, the statistical measure is prioritized. Also, when the measure is taken near a high edge value, the first term is close to zero and cancels the effect of statistical. This observation allows the following dynamic formulation for the $\tau$ argument:

$$
\tau=k \cdot \min \left(\left(\frac{\operatorname{Grad}(v(s))}{\operatorname{Grad}_{\text {mean }}}\right), 1\right)
$$

where $k \in[0,1]$ is a weighting constant. The $\operatorname{Dist}(v(s))$ function in equation (6) can be defined using the Mahalanobis distance. The Mahalanobis distance not only looks at variations (variance) between the range for one spectral band, but also at the inter-spectral level (covariance). Thus, the Mahalanobis distance defines a multi-dimensional metric space whose boundaries determine the range of variation that is acceptable for unknown samples to be classified as members of the population. This Mahalanobis distance for a sample $x$ from $\mathbf{m}$ is defined as follow:

$$
(x-\mathbf{m})^{T} C^{-1}(x-\mathbf{m})
$$

Since we assume that $x$ follows a gaussian distribution, we know that the Mahalanobis distance follow a $\chi^{2}$ distribution (Saporta, 1990) with $\mathbf{n}$ degrees of freedom ( $\mathbf{n}$ being the number of bands for the multi-spectral image). Thus, we can set a threshold value based on some confidence value ( $1 \%$ for instance). This threshold value will serve to normalize the calculated Mahalanobis distance for tested samples and also to identify outliers. Thus, the $\operatorname{Dist}(v(s))$ function is defined as follow:

$$
\operatorname{Dist}(v(s))=\frac{(v(s)-\mathrm{m})^{T} C^{-1}(v(s)-\mathrm{m})}{\text { thresholdDist }}
$$

where thresholdDist is given by the $\chi^{2}$ table value according to the dimension of the data and to the desired confidence value.

\section{FINITE ELEMENTS}

As mentioned earlier, the database vectors serve to initialize the snake using a finite elements approach. The finite elements approach offers accurate discritization of derivatives and optimizes time processing since it has fewer elements to cope with. The interested reader will find all details in Bentabet et al (2001). To summarize, the discretizing of the parametric curve $\mathrm{v}(\mathrm{s})$ into finite elements leads to an expression for each element given by:

$$
v^{e}(s)=\langle N(s)\rangle \cdot\left[V^{e}\right]
$$

where $\langle\cdots\rangle$ denotes row vectors and $[\cdots]$ matrices or column vectors. $N(s)$ is the set of interpolation curves. In our case, we used a Hermite basis functions $\left\langle N_{1}(s), N_{2}(s), \ldots, N_{m}(s)\right\rangle$ defining the form of the interpolating curve. $V^{e}$ is a two-column matrix $\left\lfloor X^{e}, Y^{e}\right\rfloor$ of control nodes inserted at regular intervals of the initial curve issued from the database. The global energy minimization is performed using the Euler Lagrange equation $\left(\nabla E_{t o t}=0\right)$. This leads to the following differential equation: 


$$
\gamma \frac{\partial v(s, t)}{\partial t}-\alpha v^{\prime \prime}(s, t)+\beta v^{\prime \prime \prime \prime}(s, t)=-\frac{\partial E_{e x t}(v(s, t))}{\partial v}
$$

where $\gamma$ controls the speed of evolution of the snake. Equation (11) is discretized using the finite elements detailed in Bentabet et al (2001). The final iterative equation is given as follow:

$$
V_{(n+1) T}^{e}=\left(I+\frac{T}{\gamma} K\right)^{-1}\left(V_{n T}^{e}-\frac{\gamma}{T} F_{n T}^{e}\right)
$$

where $I$ is the $N \times N$ identity matrix. $K$ is an $N \times N$ matrix which depends on $\alpha, \beta$ and the shape of the interpolating curves. The Hermite polynomial base determine the elements of $K . V_{n T}^{e}$ gives the location of the element nodes at iteration $n$.

\section{SNAKE PARAMETERS}

From the experience of existing works, it has been established that it is difficult to set the rigidity and the elasticity parameters of the internal energy of the snake correctly (Horritt, 1999). Indeed, they are usually fixed within a lengthy trial-and-error testing process. In this work, we estimate the rigidity parameter $\beta$ from the average curvature of the initial database vectors as follow:

$$
\beta=\beta_{\max } \cdot(1-2 \cdot \overline{c u r v})
$$

where $\beta_{\max }$ is the maximum value assigned to the rigidity parmeter, $\overline{c u r v}$ is a measure of the average curvature of the snake initialization using the segment of the database. $\beta$ is evaluated knowing that the rigidity parameter is inversely proportional to the snake curvature. This parameter setting method was proposed and is described in more detail by Bentabet et al (2001). The elasticity parameter $\alpha$ is set to a value near zero in order to allow the snake to stretch according to the external energy only. While the minimization process is running, the snake parameters are decreased in parallel in order to enable the snake to fit accurately to the high curved parts of the area borders. Multiplying them with a constant factor smaller than one ( 0.75 for instance) allows the decrease of the snake parameter values. Also, updating the control nodes of the snakes optimizes the iterative energy minimization process. Indeed, three principal operations are performed: addition of new control points as the snake expands, deletion of control points according to the local curvature of the snake and lastly, the stopping of control points when the optimal locations are reached.

\section{ALGORITHM}

The proposed algorithm takes as input the area vector database coordinates and a multi-spectral image of the same region. This algorithm can be summarized as follow:

- Perform multi-spectral edge detection;

- For every area vector to update from the database:

- Compute the statistics of the region using equation (4);

- Set the initial $\alpha$ parameter and compute the initial $\beta$ parameter using equation (13);

- Until the snake energy is not minimum:

- Compute the external force of the snake from equation (6);

- Compute the new location of the region using equation (12);

- Decrease the snakes parameters;

- If needed:

- Adjust the snake topology by re-sampling control points and/or stopping control points. 


\section{EXPERIMENTAL RESULTS}

The proposed approach was experimented on Landsat7 images using 7 bands to update features of the Canadian National Topographic Database (NTDB). The presented results in figure 1 show two kinds of patterned areas. The region 1 and 2 represent water regions that are characterized by a low variance and a high value for the gradient. In contrast, region 3 defines a clearing in a forested area. This surface is characterized by a high variance and low gradient. The flexibility of weighting between statistics and gradient allowed us to deal with these two kinds of patterned areas providing greater localization accuracy. The final snake locations are drawn over the fourth radiometric band of the Landsat image portion. To visually evaluate these results, one should consider that other radiometric bands of the image might contain complementary information that cannot be seen on the image where the results are displayed.

\section{CONCLUSION}

Our work demonstrates the importance of using snakes and multi-spectral images for updating existing spatial area information. We combined statistics and radiometry for the external energy. An efficient implementation using finite elements has been proposed providing accurate localization.

\section{REFERENCES}

Auclair-Fortier, M.F., D. Ziou, C. Armenakis and S. Wang, 2001. Automated Correction and Updating of Road Databases from High-Resolution Imagery, Canadian Journal of Remote Sensing, Vol. 27, no. 1, pp. 7689.

Bentabet, L., S. Jodouin, D. Ziou and J. Vaillancourt, 2001. Automated Updating of Road Databases from SAR Imagery: Integration of Road Databases and SAR Imagery information, Proceedings of the Fourth International Conference on Information Fusion, Vol. WeA1, pp. 3-10.

Drewniok, C., 1994. Multi-Spectral Edge Detection: Some Experiments on Data from Landsat-TM, International Journal of Remote Sensing, Vol. 15, no. 18, pp. 3743-3765.

Horritt, M.S., 1999. A statistical active contour model for SAR image segmentation, Image and Vision Computing, Vol. 17, pp. 213-224.

Kass, M., A. Witkin, and D. Terzopoulos, 1988. Snakes: Active Contour Models, International Journal of Computer Vision, Vol. 1, no. 4, pp. 321-331.

Saporta, G., 1990. Probabilité analyse de données et statistique, Édition Technip, ISBN: 2-7108-0565-0. 


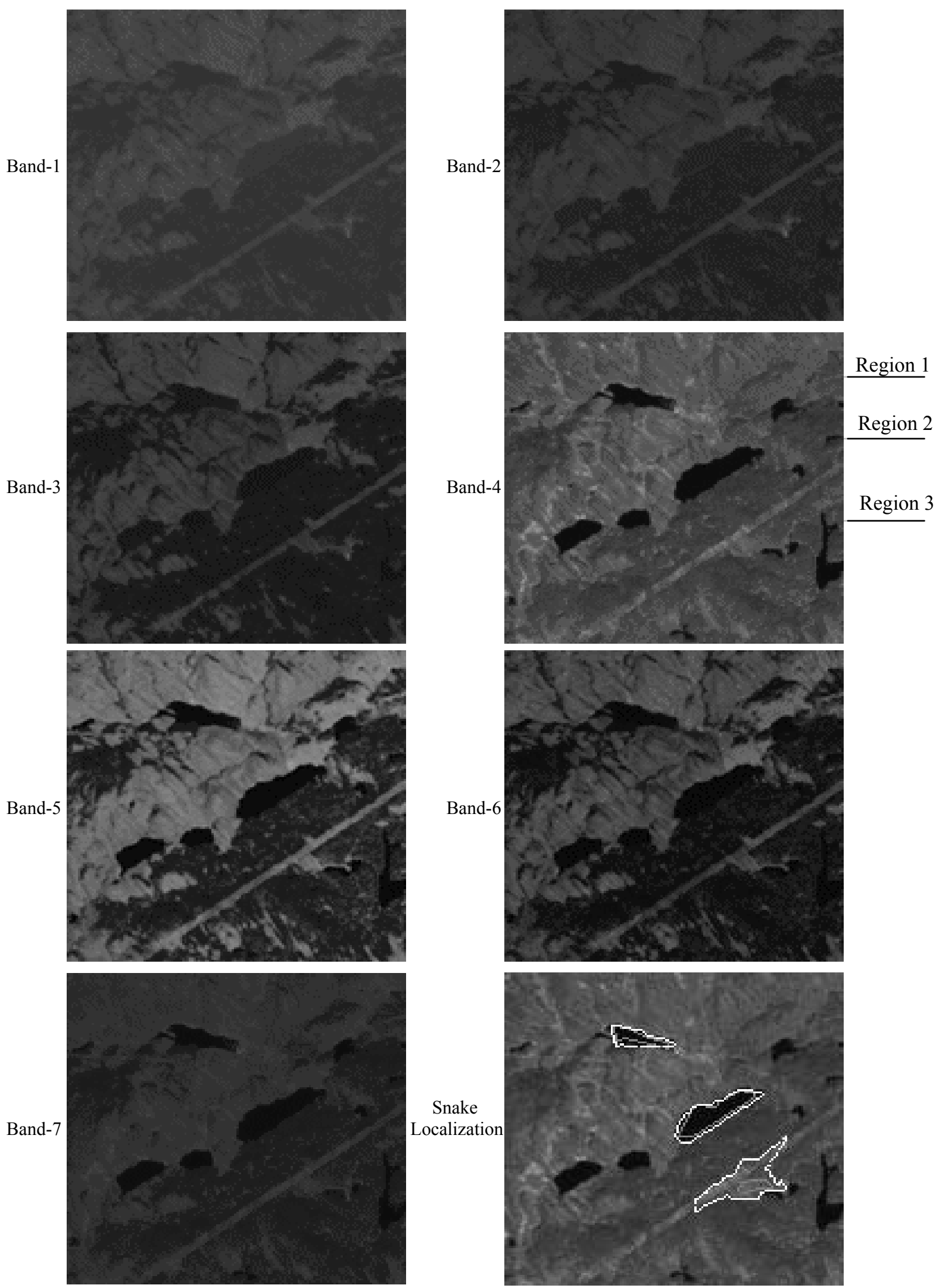

Figure 1: Experimental results with Landsat 7 images for two types of area.

(C) Departement of Natural Resources Canada. All rights reserved. 